\title{
Vancomycin-resistant Enterococcus faecium high-resolution typing by core genome multilocus sequence typing
}

\author{
Antonella Santona ${ }^{1}$, Elisa Taviani ${ }^{2}$, Massimo Deligios ${ }^{1}$, Ahmed A Al-Qahtani ${ }^{3}$, Mohammed N Al-Ahdal ${ }^{3}$, \\ Salvatore Rubino ${ }^{1}$, Bianca Paglietti ${ }^{1}$ \\ ${ }^{1}$ Department of Biomedical Sciences, University of Sassari, Sassari, Italy \\ 2 Centro de Biotecnologia, University Eduardo Mondlane (UEM), Maputo, Mozambique \\ ${ }^{3}$ Department of Infection and Immunity, King Faisal Specialist Hospital and Research Centre, Riyadh, Saudi Arabia
}

Key words: VREf typing; MLST; WGS; cgMLST.

J Infect Dev Ctries 2016; 10(10):1159-1161. doi:10.3855/jidc.9223

(Received 28 July 2016- Accepted 10 August 2016)

Copyright (C) 2016 Santona et al. This is an open-access article distributed under the Creative Commons Attribution License, which permits unrestricted use, distribution, and reproduction in any medium, provided the original work is properly cited.

Dear Editor,

In recent decades, the harmless commensal Enterococcus faecium (E. faecium) has become an important nosocomial pathogen worldwide [1]. This is mainly due to high genome plasticity, which allows $E$. faecium to acquire, by horizontal gene transfer, virulence genes and clusters, as well as antibiotic resistance determinants. Notably, the acquisition of the vancomycin resistance operons gives rise to the more worrisome vancomycin-resistant E. faecium (VREf), which is able to prompt wounds, urinary tract infections, and bacteraemia, especially in severely ill and immune-compromised patients [2], increasing length of patients' hospital stays, mortality, and healthcare costs [3].

Currently, nosocomial E. faecium clones are identified by the Multi Locus Sequence Typing (MLST) method [4], which allows the identification of specific sequence types (STs) included in the hospitalassociated clonal complex 17 (CC17) [5]. However, due to the high recombination rate in E. faecium and to the small number of MLST genes, the resolution of traditional MLST is limited.

In the past year, whole-genome analysis based on next-generation sequencing technologies has become a good alternative to the classical typing methods for nosocomial bacterial pathogens $[6,7]$.

Thanks to the increase of $E$. faecium whole-genome sequencing (WGS) data, the correct ancestral origin of CC17, previously incorrectly thought to be the sequence type ST17, now includes also ST18 and ST78 clones [8]. ST17 and ST18 represented the first wave of hospital-acquired infections in the 1980s in United States [9], while ST78, the successful hospitalassociated lineage circulating since 2005 in Europe [10] thought to have evolved from farm or pet animals [11], is now being isolated at increasing rates in hospitals in Europe, Asia, and South America [1].

Recently, a core genome MLST (cgMLST) scheme for E. faecium strains, consisting of 1,423 target genes, was developed by de Been et al., and compared by the authors to a single-nucleotide polymorphism (SNP)based approach since it allows a high-resolution tracing of E. faecium clones [12].

Here, the E. faecium cgMLST scheme within the SeqSphere+2.3 platform (Ridom GmbH, 115 Münster, Germany; http://www.ridom.de/seqsphere/), was applied to contigs obtained by WGS of 4 selected VREf clones (strains SAU1, SAU16, SAU27, and SAU28) spreading in a Saudi Arabian hospital [13], and of 17 E. faecium whole-genome sequences publicly available in GenBank (ftp://ftp.ncbi.nih.gov/genomes/) (Table 1). A total of 17 different STs were represented. cgMLST was applied with 767 targets out of the 1,423 identified in the de Been scheme, due to the poor quality of 656 targets in draft genomes.

VREf isolates obtained from the King Faisal Specialist Hospital and Research Centre (Riyadh, Saudi Arabia) were characterized in our previous studies $[13,14]$. Three isolates (SAU1, SAU16, and SAU28) were VanB type, of which the last showed a VanA phenotype, while isolate SAU27 was VanA. Additionally, the isolates were multidrug resistant and carried the IS16 element [13], which is related to an 
enhanced potential of E. faecium for nosocomial transmission and is considered a molecular screening marker for hospital-associated strains. By MLST, VREf isolates were assigned to sequence types ST117, ST795, ST80, and ST546, all belonging to CC17. Moreover, the comparative eBURST analysis with the STs of the entire E. faecium MLST database showed that both ST795 and ST80 derived from ST117, and that ST546 derived from ST17 [13]. Here, the cgMLST analysis confirmed the genetic relatedness of isolates SAU1 (ST117) and its related mutant (SLV) SAU28 (ST795) (Figure 1). It also revealed the correct origin of the epidemic clone ST80 (SAU27) grouped here with its DLV ST18 (Figure 1) and not with its SLV ST117, as previously improperly predicted by the traditional MLST coupled with eBURSTv3 analysis [13]. Moreover, the UPGMA phylogenetic three showed that the ST546 clone, like ST80, clustered with ST18, suggesting the latter and not ST17 was the ancestor for both sequence types, and that ST546 probably evolved from ST80 during hospital adaptation.

Comparative genome analysis revealed that the Saudi Arabian VREf isolates were virulent and dangerous. They possessed a high level of homology with virulence genes and gene clusters recognized to be specific for hospital-associated E. faecium (Table 2), including the genomic island GI. This provides a competitive advantage over the indigenous E. faecium microbiota [15], as previously demonstrated for isolate SAU28 [13].

Furthermore, isolates SAU1 and SAU16, belonging to different STs, carried the same $\mathrm{VanB}_{2}$ operon with $99.8 \%$ homology with the Tn1549 of strain TSGH1 (AF310956), suggesting lateral exchange of this genetic element among the isolates. The VanA type strain (SAU27) carried the Tn1546 that showed 100\% sequence homology with the transposon carried by the E. faecium Efm32007 strain (KR349520).

In conclusion, the cgMLST scheme was useful in identifying the correct common origin of the micro-

Figure 1. UPGMA phylogenetic tree of 4 selected VREf clones and of $17 \mathrm{E}$. faecium whole-genome sequences publicly available in GenBank, generated by the SeqSphere +2.3 based on 767 targets.

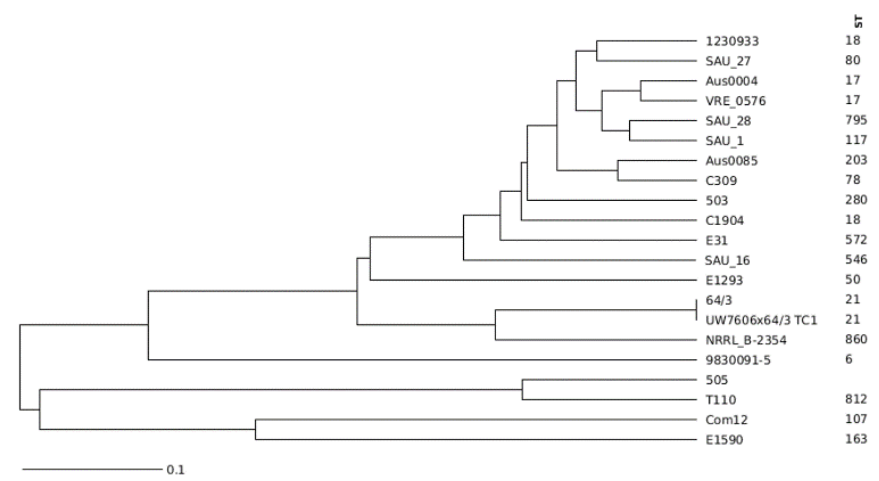

UPGMA tree for 21 Samples based on 767 columns, no missing values, \% columns difference

Table 1. E. faecium strains used in cgMLST analysis.

\begin{tabular}{|c|c|c|c|c|c|}
\hline Strain & $S T$ & $\begin{array}{l}\text { Chromosome/W } \\
\text { GS Accession }\end{array}$ & Size $(M b)$ & Institution & $\begin{array}{r}\text { Release } \\
\text { Date }\end{array}$ \\
\hline SAU1 & ST117 & This study & $2,889,771$ & KFSHRC (Riyadh) & -- \\
\hline SAU16 & ST546 & This study & $2,774,380$ & KFSHRC (Riyadh) & $06 / 26 / 2016$ \\
\hline SAU27 & ST80 & This study & $2,922,922$ & KFSHRC (Riyadh) & $06 / 26 / 2016$ \\
\hline SAU28 & ST795 & This study & $3,062,080$ & KFSHRC (Riyadh) & $06 / 26 / 2016$ \\
\hline Aus0004 & ST17 & NC_017022.1 & $3,019,779$ & University of Melbourne & $03 / 01 / 2012$ \\
\hline VRE0576 & ST17 & JAAK 0000000 & $3,132,850$ & Swedish Institute for Communicable Disease Control & $14 / 04 / 2014$ \\
\hline Aus0085 & ST203 & NC_021994.1 & $3,239,279$ & University Of Melbourne & $08 / 06 / 2013$ \\
\hline $\mathrm{T} 110$ & ST812 & NZ_CP006030.1 & $2,737,960$ & SRM University & $08 / 04 / 2014$ \\
\hline 1230933 & ST18 & ACAS01 & $3,140,779$ & Broad Institute & $06 / 15 / 2009$ \\
\hline 503 & ST280 & AMBN01 & $2,746,730$ & Washington University & $09 / 04 / 2012$ \\
\hline C1904 & ST18 & AMBD01 & $2,855,420$ & Washington University & $09 / 04 / 2012$ \\
\hline EnGen0006/E1293 & ST50 & AHWU01 & $2,868,639$ & Broad Institute & $05 / 03 / 2013$ \\
\hline E31 & ST572 & JYCO01 & $3,048,470$ & Beijing Chao-Yang Hospital, Capital Medical Univ. & $03 / 12 / 2015$ \\
\hline C309 & ST78 & AJTW01 & $3,102,430$ & Shanghai Chinese Academy of Sciences & $12 / 24 / 2012$ \\
\hline 64/3 VRE0576 & ST21 & СР012522.1 & $2,572,329$ & Robert Koch Institute & $09 / 24 / 2015$ \\
\hline UW7606x64/3 TC1 & ST21 & СР013009.1 & 2.753350019 & Robert Koch Institute & $10 / 26 / 2015$ \\
\hline NRRL B-2354 & ST860 & NC_020207.1 & 2.849889994 & University of California, Davis & $02 / 04 / 2013$ \\
\hline EnGen0138/9830091-5 & ST6 & AITC01 & 2.637219906 & Broad Institute & $05 / 03 / 2013$ \\
\hline 505 & - & AMBL01 & 2.619859934 & Washington University & $09 / 04 / 2012$ \\
\hline Com12 & ST107 & $\mathrm{ACBC} 01$ & 2.71374011 & Broad Institute & $05 / 20 / 2009$ \\
\hline EnGen0003/E1590 & ST163 & $\mathrm{AHXC01}$ & 3.021650076 & Broad Institute & $12 / 17 / 2012$ \\
\hline
\end{tabular}


Table 2. Homology of hospital-associated genes and gene clusters present in VREf. Percent of identity to the reference sequence is indicated for each gene and gene cluster.

\begin{tabular}{|c|c|c|c|c|c|}
\hline Gene/gene clusters & Accession no. & SAU1 & SAU16 & SAU27 & SAU28 \\
\hline $\operatorname{Tn} 1546$ & KR349520 & - & - & 100 & - \\
\hline $\operatorname{Tn} 1549$ & AF310956 & 99.8 & 99.8 & - & 99.5 \\
\hline efaAfm & FJ609170.1 & 94 & 94 & 94 & 94 \\
\hline sgrAadhesin & EFAU085 01549 & 100 & 99.9 & 100 & 99.9 \\
\hline $\mathrm{acm}$ & EFAU08502356 & 99 & 100 & 100 & 100 \\
\hline$e s p$ & EFAU004 02750 & 100 & 99.6 & 100 & $>90$ \\
\hline Genomic island (GI) & AAAK03000019 & $>90$ & $>90$ & $>90$ & $>90$ \\
\hline IS16/IS256 & EFAU004 02683 & 100 & 100 & 100 & 100 \\
\hline Pilin 1 gene cluster & EU90969 & 100 & 99.5 & 91 & 99.1 \\
\hline Pilin 3 gene cluster & EU909696 & 100 & 100 & 100 & 100 \\
\hline
\end{tabular}

epidemic of ST80 and ST546, undetected by traditional MLST coupled with eBURSTv3 analysis, and to highlight a high genetic recombination between $\mathrm{CC} 17$ clones spreading within the hospital. It was shown to be a suitable tool to carefully trace E. faecium clones and it would therefore be very helpful in hospital surveillance.

WGS and the cgMLST scheme allow for the exploration of virulence genes, antibiotic resistance mechanisms, and other important factors concordantly with molecular epidemiology investigation of bacterial outbreaks; they have a great ability to define clone relationships, thus significantly impacting the practice of infection control.

\section{References}

1. Willems RJL, Top J, Van Santen M, Robinson DA, Coque TM, Baquero F, Grundmann H, Bonten MJ (2005) Global spread of vancomycin-resistant Enterococcus faecium from distinct nosocomial genetic complex. Emerg Infect Dis 11: 821-828.

2. Top J, Willems R, Bonten M (2008) Emergence of CC17 Enterococcus faecium: From commensal to hospital-adapted pathogen. FEMS Immunol Med Microbiol 52: 297-308.

3. Cheah AL, Spelman T, Liew D, Peel T, Howden BP, Spelman D, Grayson ML, Nation RL, Kong DC (2013) Enterococcal bacteraemia: factors influencing mortality, length of stay and costs of hospitalization. Clin Microbiol Infect 19: 384.

4. Homan WL, Tribe D, Poznanski S, Li M, Hogg G, Spalburg E, Van Embden JD, Willems RJ (2002) Multilocus Sequence Typing Scheme for Enterococcus faecium Multilocus Sequence Typing Scheme for Enterococcus faecium. J Clin Microbiol 40: 1963-1971.

5. Leavis HL, Bonten MJM, Willems RJL (2006) Identification of high-risk enterococcal clonal complexes: global dispersion and antibiotic resistance. Curr Opin Microbiol 9: 454-460.

6. Gilmour MW, Graham M, Reimer A, Van Domselaar G (2013) Public health genomics and the new molecular epidemiology of bacterial pathogens. Public Health Genomics 16: 25-30.

7. Kwong JC, McCallum N, Sintchenko V, Howden BP (2015) Whole genome sequencing in clinical and public health microbiology. Pathology 47: 199-210.

8. Willems RJL, Top J, van Schaik W, Leavis H, Bonten M, Sirén J, Hanage WP, Corander J (2012) Restricted gene flow among hospital subpopulations of Enterococcus faecium. MBio 3: 110.
9. Galloway-Peña JR, Nallapareddy SR, Arias CA, Eliopoulos GM, Murray BE (2009) Analysis of clonality and antibiotic resistance among early clinical isolates of Enterococcus faecium in the United States. J Infect Dis 200: 1566-1573.

10. Klare I, Konstabel C, Mueller-Bertling S, Werner G, Strommenger B, Kettlitz C, Borgmann S, Schulte B, Jonas D, Serr A, Fahr AM, Eigner U, Witte W (2005) Spread of ampicillin/vancomycin-resistant Enterococcus faecium of the epidemic-virulent clonal complex-17 carrying the genes esp and hyl in German hospitals. Eur J Clin Microbiol Infect Dis 24: 815-825.

11. Hammerum AM (2012) Enterococci of animal origin and their significance for public health. Clin Microbiol Infect 18: 619625.

12. De Been M, Pinholt M, Top J, Bletz S, Mellmann A, van Schaik W, Brouwer E, Rogers M, Kraat Y, Bonten M, Corander J, Westh H, Harmsen D, Willems RJ (2015) Core genome multilocus sequence typing scheme for highresolution typing of Enterococcus faecium. J Clin Microbiol 53: 3788-3797.

13. Santona A, Paglietti B, Al-Qahtani AA, Bohol MFF, Senok A, Deligios M, Rubino S, Al-Ahdal MN (2014) Novel type of VanB2 teicoplanin-resistant hospital-associated Enterococcus faecium. Int J Antimicrob Agents 44: 156-159.

14. Al-Ahdal MN, Abozaid SM, Al-Shammary HF, Bohol MF, AlThawadi SI, Al-Jaberi AA, Senok AC, Shibl AM, Al-Qahtani AA (2012) Characterization of Enterococcus faeciumisolates and first report of vanB phenotype-vanA genotype incongruence in the Middle East. Eur J Clin Microbiol Infect Dis 31: 3223-3229.

15. Heikens E, Van Schaik W, Leavis HL, Bonten MJM, Willems RJL (2008) Identification of a novel genomic island specific to hospital-acquired clonal complex 17 Enterococcus faecium isolates. Appl Environ Microbiol 74: 7094-7097.

\section{Corresponding author}

Antonella Santona

Department of Biomedical Sciences, University of Sassari, Viale San Pietro 43/b, 07100 Sassari, Italy

Phone: +39079228300

Fax: +39079212345

Email: asantona@uniss.it

Conflict of interests: No conflict of interests is declared. 